\title{
THE EIGENVALUE BEHAVIOR OF CERTAIN CONVOLUTION EQUATIONS
}

BY

H. J. LANDAU

Introduction. In a series of papers [3], [4], [6], we studied the relationship between two closed subspaces of $L^{2}(-\infty, \infty)$ : the subspace $\mathscr{D}_{T}$ of all $f \in L^{2}$ supported in $|t|<T / 2$ and the subspace $\mathscr{B}_{\Omega}$ of all $f \in L^{2}$ whose Fourier transforms are supported in $|\omega|<\Omega / 2$. We showed that several questions about $\mathscr{D}_{T}$ and $\mathscr{D}_{\Omega}$ could be answered in terms of the eigenvalues of the operator $B_{\Omega} D_{T} B_{\Omega}$, where $B_{\Omega}$ and $D_{T}$ are the projections onto $\mathscr{B}_{\Omega}$ and $\mathscr{D}_{T}$ respectively; this operator may be written as a finite convolution. Apart from this application, interpretable as describing the way in which the energy of a function of $L^{2}$ can be distributed over time and over frequency, the behavior of these eigenvalues is interesting because it differs markedly from that established by $\mathrm{H}$. Widom [7] for the class of finite convolutions with $L^{1}$ kernels whose Fourier transforms have an absolute maximum at the origin.

By a change of variable, the eigenvalues of $B_{\Omega} D_{T} B_{\Omega}$ may be seen to depend on the parameter $c=\Omega T / 2 \pi$, rather than on $\Omega$ and $T$ separately; we may write their equation explicitly as

$$
\lambda_{n}(c) \phi_{n}^{(c)}(t)=\frac{1}{\pi} \int_{-c / 2}^{c / 2} \frac{\sin \pi(t-x)}{t-x} \phi_{n}^{(c)}(x) d x, \quad n=0,1,2, \cdots,
$$

and we suppose that $\lambda_{0} \geqq \lambda_{1} \geqq \ldots$. For any fixed $c$, the $\lambda_{n}(c), n=0,1, \cdots$, form a positive sequence bounded away from 1 and approaching 0 at a rate in $n$ greater than $(c e / n)^{2 n}$ [D. Slepian, unpublished]. For any fixed $n$, the eigenvalue $\lambda_{n}(c)$ approaches 1 exponentially in $c$ [2]. In [4] we proved, however, that $\lambda_{[c]+1}(c)$ is bounded away from 1 independently of $c$, and interpreted this to imply that the set of functions in $\mathscr{B}_{\Omega}$ whose energy is concentrated in $|t|<T / 2$ has, in a well-defined sense, approximate dimension bounded by $[\Omega T / 2 \pi]\left({ }^{1}\right)$. We also showed that $\lambda_{[c]-1}(c)$ is bounded away from 0 independently of $c$.

The analogous questions for the case where the intervals $|t|<T / 2$ and $|\omega|<\Omega / 2$ are replaced by more general sets $T^{\prime}$ and $\Omega^{\prime}$ have not been studied. Indeed, most of the methods developed to deal with $\mathscr{B}_{\Omega}$ are not applicable to $\mathscr{B}_{\Omega^{\prime}}$, and very little is known about it. Here we give another, simpler, proof that $\lambda_{[c]+1}(c)$ and $\lambda_{[c]-1}(c)$ are bounded away from 1 and 0 respectively,

Received by the editors December 2, 1963.

( $\left.{ }^{1}\right)[x]$ denotes the largest integer less than or equal to $x$. 
independently of $c$. Our method improves considerably on the bound of [4], but its most interesting feature is its extendability to the case that $T^{\prime}$ and $\Omega^{\prime}$ are each finite unions of intervals.

AcKnowledgements. I am greatly indebted to H. O. Pollak, D. Slepian, and L. J. Wallen for many interesting conversations.

Preliminaries. We consider the Hilbert space $L^{2}(-\infty, \infty)$ with the usual definition of the scalar product

$$
(f, g)=\int_{-\infty}^{\infty} f(t) \overline{g(t)} d t,
$$

and denote by $F(\omega)$ the Fourier transform of $f(t) \in L^{2}$,

$$
F(\omega)=(2 \pi)^{-1 / 2} \int_{-\infty}^{\infty} f(t) e^{-i \omega t} d t .
$$

If $S$ is an open subset of the real line, we single out two subspaces of $L^{2}$ :

$$
\begin{aligned}
& \mathscr{D}(S)=\left\{f \in L^{2} \mid f(t) \equiv 0, t \notin S\right\}, \\
& \mathscr{D}(S)=\left\{f \in L^{2} \mid F(\omega) \equiv 0, \omega \notin S\right\} .
\end{aligned}
$$

If $S$ is a bounded set, and $f \in \mathscr{B}(S)$, writing $f(t)$ as the inverse transform of $F(\omega)$ exhibits $f$ as the restriction to the reals of an entire function $f(t+i u)$ of exponential type. If $\|f\|=1$, Schwarz's inequality and Parseval's theorem applied to this representation show that $f(t+i u)$ is bounded in any given horizontal strip, by a constant depending only on the strip.

$\mathscr{D}(S)$ and $\mathscr{B}(S)$ are closed, since the Fourier transform preserves the norm. Let $D_{S}$ and $B_{S}$ denote the orthogonal projection operators of $L^{2}$ onto $\mathscr{D}(S)$ and $\mathscr{B}(S)$ respectively; their concrete representation is

$$
\begin{aligned}
& D_{S} f(t)=\chi_{S}(t) f(t), \\
& B_{S} f(t)=(2 \pi)^{-1 / 2} \int_{-\infty}^{\infty} \chi_{S}(\omega) F(\omega) e^{i \omega t} d \omega,
\end{aligned}
$$

where $\chi_{S}(u)$ is the characteristic function of $S$, i.e.,

$$
\chi_{S}(u)= \begin{cases}1, & u \in S \\ 0, & u \notin S .\end{cases}
$$

If $S$ and $S^{\prime}$ are two open sets, the operator $B_{S} D_{S^{\prime}} B_{S}$ is bounded by 1 , selfadjoint, and positive. If $S$ and $S^{\prime}$ have finite measure we may write $B_{S} D_{S^{\prime}}$ explicitly as

$$
B_{S} D_{S^{\prime}} \phi=(2 \pi)^{-1 / 2} \int_{-\infty}^{\infty} \chi_{S^{\prime}}(u) h(t-u) \phi(u) d u,
$$

where the Fourier transform of $h$ coincides with $\chi_{S}(\omega)$. Since, by Parseval's theorem; 


$$
\int_{-\infty}^{\infty} d u \int_{-\infty}^{\infty} d t\left|\chi_{S^{\prime}}(u) h(t-u)\right|^{2}=\int_{-\infty}^{\infty}\left|\chi_{S^{\prime}}(u)\right|^{2} d u \int_{-\infty}^{\infty}\left|\chi_{S}(\omega)\right|^{2} d \omega<\infty,
$$

the operator $B_{S} D_{S^{\prime}}$ is completely continuous $[5$, p. 159], and hence so is $B_{S} D_{S^{\prime}} B_{S}$. Consequently $\left[5\right.$, p. 233] the spectrum of $B_{S} D_{S^{\prime}} B_{S}$ consists of isolated positive eigenvalues, bounded by 1 and accumulating at zero. In concrete form, the eigenvalue equation is a convolution:

$$
\lambda_{n} \phi_{n}(t)=(2 \pi)^{-1 / 2} \int_{x \in S^{\prime}} \phi_{n}(x) h(t-x) d x .
$$

Results.

THeOREM 1. Let $P$ and $Q$ be intervals of lengths $W$ and $T$ respectively, and let $c=W T / 2 \pi$. Let $\lambda_{0}, \lambda_{1}, \cdots$ be the eigenvalues of $B_{P} D_{Q} B_{P}$, arranged in nonincreasing order. Then $\lambda_{[e]+1} \leqq .6$.

Proof. By a change of scale on the sets $P$ and $Q$ which does not alter $c$ or the eigenvalues $\lambda_{i}$ we may normalize the problem so that $W=2 \pi, T=c$, $P$ coincides with $|\omega|<\pi$ and $Q$ with $-1 / 2<t<c-1 / 2$. To simplify notation we henceforth drop the subscripts on $B_{P}$ and $D_{Q}$.

The Weyl-Courant lemma $[5$, p. 238] asserts that

$$
\lambda_{[c]+1} \leqq \sup _{\left(f, \psi_{i}\right)=0} \frac{(B D B f, f)}{\|f\|^{2}},
$$

where $\psi_{i}, i=0, \cdots,[c]$, are any $[c]+1$ functions of $L^{2}$. Since $B$ and $D$ are orthogonal projections, they are self-adjoint and idempotent operators, so that

$$
(B D B f, f)=\left(D^{2} B f, B f\right)=\|D B f\|^{2} .
$$

Furthermore $\|f\|^{2} \geqq\|B f\|^{2}$, with equality equivalent to $f=B f$, that is to $f \in \mathscr{B}(P)$. Consequently we may rewrite (1) as

$$
\lambda_{[c]+1} \leqq \sup _{f \in \mathscr{B}(P) ;\left(f, \psi_{i}\right)=0} \frac{\|D f\|^{2}}{\|f\|^{2}}, \quad i=0, \cdots,[c] .
$$

Let $h(t) \in L^{2}$ vanish for $|t| \geqq 1 / 2$ and let its Fourier transform $H(\omega)$ satisfy

$$
|H(\omega)| \geqq 1, \quad|\omega| \leqq \pi .
$$

Now given $f \in \mathscr{B}(P)$, we consider the function

(4) $g(x)=(2 \pi)^{-1 / 2} \int_{-\infty}^{\infty} f(t) h(x-t) d t=(2 \pi)^{-1 / 2} \int_{|t-x|<1 / 2} f(t) h(x-t) d t$,

whose Fourier transform is $F(\omega) H(\omega)$. Since $f \in \mathscr{B}(P)$, the function $F(\omega)$ 
vanishes for $|\omega| \geqq \pi$, and $H(\omega)$ is bounded, so that $F(\omega) H(\omega) \in L^{2}(-\pi, \pi)$. Let $a_{k}$ be the $(-k)$ th coefficient of the Fourier series expansion of $F(\omega) H(\omega)$ in $|\omega|<\pi$. By definition

(5) $a_{k}=(2 \pi)^{-1 / 2} \int_{-\pi}^{\pi} F(\omega) H(\omega) e^{i k \omega} d \omega=(2 \pi)^{-1 / 2} \int_{-\infty}^{\infty} F(\omega) H(\omega) e^{i k \omega} d \omega=g(k)$,

whence using Parseval's theorem and (3)

(6) $\|f\|^{2}=\int_{-\pi}^{\pi}|F(\omega)|^{2} d \omega \leqq \int_{-\pi}^{\pi}|F(\omega) H(\omega)|^{2} d \omega=\sum_{-\infty}^{\infty}\left|a_{k}\right|^{2}=\sum_{-\infty}^{\infty}|g(k)|^{2}$.

Next let us in (2) set

$$
\psi_{i}(t)=\overline{h(i-t)}, \quad i=0,1, \cdots,[c] .
$$

By (4) the conditions $\left(f, \psi_{i}\right)=0$ are equivalent to $g(k)=0$ for $k=0, \ldots,[c]$, so that from (6)

$$
\|f\|^{2} \leqq \sum_{k<0 ; k>|c|}|g(k)|^{2} .
$$

Schwarz's inequality applied to (4) yields

$$
|g(k)|^{2} \leqq \frac{\|h\|^{2}}{2 \pi} \int_{|t-k|<1 / 2}|f(t)|^{2} d t,
$$

and the intervals $|t-k|<1 / 2$ with $k<0$ and $k>[c]$ all lie outside $Q$. Hence, combining (8) and (9) gives

$$
2 \pi\|f\|^{2} \leqq\|h\|^{2} \int_{t \notin Q}|f(t)|^{2} d t=\|h\|^{2}\left[\|f\|^{2}-\|D f\|^{2}\right],
$$

whence

$$
\|D f\|^{2} /\|f\|^{2} \leqq 1-2 \pi /\|h\|^{2} .
$$

Letting $h(t)=\pi(\pi / 2)^{1 / 2}$ in $|t| \leqq 1 / 2$ and 0 elsewhere, (2) and (11) prove

$$
\lambda_{[c]+1} \leqq 1-4 / \pi^{2}<.6 \text {. }
$$

Theorem 1 is established.

H. O. Pollak has shown that, as in [4], the argument of Theorem 1 can serve as a basis for establishing a lower bound independent of $c$ for $\lambda_{|c|-1}$.

Theorem 2 (H. O. Pollak). Under the hypotheses of Theorem $1, \lambda_{[c]-1} \geqq .4$.

Proof. A simple consequence of the Weyl-Courant lemma is

$$
\lambda_{|c|-1} \geqq \inf _{f \in S \mid c]} \frac{(B D B f, f)}{\|f\|^{2}},
$$


where $S_{[c]}$ is any [c]-dimensional subspace of $L^{2}$. If we choose $S_{[c]}$ to be a subspace of $\mathscr{B}(P)$ we may, following Theorem 1 , rewrite (12) as

$$
\lambda_{[c]-1} \geqq \inf _{f \in \not(P) ; f \in S_{[c]}} \frac{\|D f\|^{2}}{\|f\|^{2}} .
$$

Now with the function $h(t)$ of (3), we let $S_{[c]} \subset \mathscr{B}(P)$ be the subspace spanned by the $[c]$ (independent) members of $\mathscr{B}(P)$ whose Fourier transforms are $(2 \pi)^{-1 / 2} e^{-i k \omega} / H(\omega)$ on $|\omega| \leqq \pi$, with $k=0, \ldots,[c]-1$. Then by definition, if $f \in S_{|c|}$, its Fourier transform $F(\omega)$ satisfies

$$
H(\omega) F(\omega)=\sum_{k=0}^{|c|-1} b_{k}(2 \pi)^{-1 / 2} e^{-i k \omega}, \quad|\omega| \leqq \pi,
$$

so that, as in Theorem 1, we have $H(\omega) F(\omega)$ written as a Fourier series. Then, as in (5), (6), and (9)

$$
\begin{gathered}
\|f\|^{2} \leqq \sum_{0}^{\mid c j-1}\left|b_{k}\right|^{2} \\
\left|b_{k}\right|^{2}=\left|(2 \pi)^{-1 / 2} \int_{|t-k|<1 / 2} f(t) h(k-t) d t\right|^{2} \leqq \frac{\|h\|^{2}}{2 \pi} \int_{|t-k| \leqq 1 / 2}|f(t)|^{2} d t,
\end{gathered}
$$

but now the intervals $|t-k| \leqq 1 / 2$ for $k=0, \cdots,[c]-1$ all lie inside $Q$. Hence, combining (15) and (16) gives

$$
2 \pi\|f\|^{2} \leqq\|h\|^{2} \int_{t \in Q}|f(t)|^{2} d t=\|h\|^{2}\|D f\|^{2},
$$

which implies $\|D f\|^{2} /\|f\|^{2} \geqq 2 \pi /\|h\|^{2}$ for all $f \in S_{|c|}$. By (13), choosing the $h(t)$ of Theorem 1 ,

$$
\lambda_{|c|-1} \geqq .4 .
$$

Theorem 2 is established.

B. F. Logan has proved [to appear] that, by proper choice of $h(t)$, the bounds of Theorems 1 and 2 can be improved to .5; together with Lemma 2 this implies that $\lim _{c \rightarrow \infty} \lambda_{|c|}(c)=1 / 2$. We showed in [4] that the change in size of $\lambda_{k}$ from near 1 to near 0 occurs in a strip around $k=[c]$ which grows no faster than $\log c$ but also does not remain bounded. Thus the description of the eigenvalues seems fairly complete.

Theorems 1 and 2 possess extensions to the case where the sets $P$ and $Q$ are finite unions of intervals.

LEMma 1. Let $S$ consist of the union of $m$ disjoint intervals and have total measure $M$. Then

a. the number $N(S)$ of integers $k$ for which the interval $|k-t|<1 / 2$ inter- 
sects $S$ does not exceed $[M]+2 m$;

b. the number $N^{\prime}(S)$ of integers $k$ for which the interval $|k-t|<1 / 2$ is contained in $S$ exceeds $M-2 m$.

Proof. If $S$ is a single interval, let it coincide with $\alpha<t<M+\alpha$, let $k_{1}$ be the least integer with $k_{1}+1 / 2>\alpha$ and $k_{2}$ the largest integer with $k_{2}-1 / 2<M+\alpha$. Then $N(S)=k_{2}-k_{1}+1<M+2$. Now if $S$ is the union of disjoint intervals $S_{i}$ of measures $m_{i}$, with $i=1, \cdots, m$, then $N(S)$ $\leqq \sum_{i} N\left(S_{i}\right)<\sum_{i}\left\{M_{i}+2\right\}=M+2 m$, consequently $N(S) \leqq[M]+2 m$.

Similarly, when $S$ is a single interval, let $k_{1}^{\prime}$ be the largest integer with $k_{1}^{\prime}-1 / 2<\alpha$ and $k_{2}^{\prime}$ the least integer with $k_{2}^{\prime}+1 / 2>M+\alpha$. Then $N^{\prime}(S)=k_{2}^{\prime}-k_{1}^{\prime}-1>M-2$, and if $S$ is the union of $m$ disjoint intervals $N^{\prime}(S)=\sum_{i} N^{\prime}\left(S_{i}\right)>\sum_{i}\left\{M_{i}-2\right\}=M-2 m$. Lemma 1 is established.

Corollary 1. Let one of the sets $P$ and $Q$ of measures $W$ and $T$ respectively, be a single interval, and the other be the union of $m$ disjoint intervals. With $c$ and $\lambda_{n}$ defined as in Theorem $1, \lambda_{|c|+2 m}<.6$ and $\lambda_{|c|-2 m}>.4$.

Proof. The spectra of $B D B$ and $D B D$ are identical. For if $B D B \phi=\lambda B \phi$, we apply $D$ to both sides and use the idempotency of $D$ to obtain $D B D(D B \phi)$ $=\lambda(D B \phi)$, and conversely. Consequently, in the proof of Theorems 1 and 2 , the roles of $t$ and $\omega$ are interchangeable. We may accordingly suppose that $P$ consists of a single interval, which we normalize as before to coincide with $|\omega|<\pi$, whereupon $Q$ becomes the union of $m$ disjoint intervals of total measure $c$. Let $N(Q)$ and $N^{\prime}(Q)$ be as in Lemma 1. We may now in the proof of Theorem 1 replace $[c]+1$ by $[c]+2 m$ in (1) and argue without further change until (7), where we choose $\psi_{i}(t)=\overline{h(i-t)}$ for those $i$ counted by $N(Q)$. By Lemma 1 , the number of functions so obtained does not exceed $[c]+2 m$, inequality (10) follows as before, and we may repeat the rest of the argument to show that $\lambda_{|c|+2 m}<.6$. Similarly, in the proof of Theorem 2 we replace $[c]-1$ by $[c]-2 m$ in (12) and $S_{[c]}$ by the subspace $S$ of $\mathscr{B}(P)$ spanned by the functions whose Fourier transforms on $|\omega| \leqq \pi$ are $(2 \pi)^{-1 / 2} e^{-i k \omega} / H(\omega)$ for those $k$ counted by $N^{\prime}(Q)$. By Lemma 1 , the number of functions so obtained exceeds $c-2 m$ and, being an integer, is no smaller than $[c]-2 m+1$. Hence $S$ has dimension at least $[c]-2 m+1$, and we may repeat the rest of the argument to show that $\lambda_{[c]-2 m}>.4$. Corollary 1 is established.

Theorem 3. Let $P$ and $Q$ be unions of $p$ and $q$ disjoint intervals of total measure $W$ and $T$ respectively. Let $c$ and $\lambda_{n}$ be defined as in Theorem 1 . Then $\lambda_{[c]+2 p q} \leqq J<1$, where $J$ is a constant depending only on $P$ (suitably normalized) but not on $Q$.

Proof. We may again suppose $W=2 \pi$ and $T=c$, since this may always 
be achieved by a change of scale on the sets $P$ and $Q$ which does not alter $c, p, q$ or the eigenvalues $\lambda_{n}$, and we drop the subscripts of $B_{P}$ and $D_{Q}$. Let us denote by $\sigma_{1}, \cdots, \sigma_{p}$ the disjoint intervals comprising $P$, let $2 \pi l_{N}$ be the length of $\sigma_{N}$, and $\chi_{N}(\omega)$ be its characteristic function. Let $h_{N}(t)$ be a function of $L^{2}$ which vanishes for $|t| \geqq 1 / 2 l_{N}$ and whose Fourier transform $H_{N}(\omega)$ satisfies

$$
\left|H_{N}(\omega)-\chi_{N}(\omega)\right|<\epsilon, \quad \omega \in P,
$$

with $\epsilon=\left\{32 p^{2}+16 \sum_{N}\left[1 / l_{N}\right]\right\}^{-1 / 2}$. Such an $h_{N}(t)$ exists, since $\chi_{N}(\omega)$ is continuous on $P$, and Fourier transforms of functions vanishing outside any fixed interval are uniformly dense in continuous functions on any compact set. Let $\gamma_{N}=\left\|h_{N}\right\|^{2}$.

As in Theorem 1, we will base our proof on the Weyl-Courant lemma, which asserts that

$$
\lambda_{[c]+2 p q} \leqq \sup _{f \in \mathscr{B}(P) ;\left(f, \psi_{i}\right)=0} \frac{\|D f\|^{2}}{\|f\|^{2}},
$$

where $\psi_{i}, i=1, \cdots,[c]+2 p q$, are any $[c]+2 p q$ functions of $L^{2}$. Now given $f \in \mathscr{B}(P)$ with Fourier transform $F(\omega)$ we consider the function

(20) $g_{N}(x)=(2 \pi)^{-1 / 2} \int_{-\infty}^{\infty} f(t) h_{N}(x-t) d t=(2 \pi)^{-1 / 2} \int_{|t-x|<1 / 2_{N}} f(t) h_{N}(x-t) d t$, whose Fourier transform is $F(\omega) H_{N}(\omega)$. By definition and a change of variable

$\left(l_{N}\right)^{-1 / 2} g_{N}\left(k / l_{N}\right)=\left(2 \pi l_{N}\right)^{-1 / 2} \int_{P} F(\omega) H_{N}(\omega) \exp \left(i \omega k / l_{N}\right) d \omega$

$$
\begin{aligned}
& =\left(2 \pi l_{N}\right)^{-1 / 2} \int_{P} \sum_{r} \chi_{N}\left(\omega+r 2 \pi l_{N}\right) F(\omega) H_{N}(\omega) \exp \left(i \omega k / l_{N}\right) d \omega \\
& =\left(2 \pi l_{N}\right)^{-1 / 2} \int_{\sigma_{N}}\left\{\sum_{r} F\left(\omega-r 2 \pi l_{N}\right) H_{N}\left(\omega-r 2 \pi l_{N}\right)\right\} \exp \left(i \omega k / l_{N}\right) d \omega \\
& =\int_{\sigma_{N}}\left\{F(\omega)+R_{N}(\omega)\right\}\left(2 \pi l_{N}\right)^{-1 / 2} \exp \left(i \omega k / l_{N}\right) d \omega,
\end{aligned}
$$

where

$$
R_{N}(\omega)=\sum_{r} F\left(\omega-r 2 \pi l_{N}\right)\left\{H_{N}\left(\omega-r 2 \pi l_{N}\right)-\chi_{N}\left(\omega-r 2 \pi l_{N}\right)\right\}, \quad \omega \in \sigma_{N} .
$$

Since the functions $\left(2 \pi l_{N}\right)^{-1 / 2} \exp \left(i \omega k / l_{N}\right)$ form a complete orthonormal set on $\sigma_{N},(21)$ implies 


$$
l_{N}^{-1} \sum_{k=-\infty}^{\infty}\left|g_{N}\left(\frac{k}{l_{N}}\right)\right|^{2}=\int_{\sigma_{N}}\left|F(\omega)+R_{N}(\omega)\right|^{2} d \omega .
$$

To estimate the size of $R_{N}(\omega)$ on $\sigma_{N}$, we observe that the summands which do not vanish identically on $\sigma_{N}$ correspond to those $r$ for which the translate of $\sigma_{N}$ by $r 2 \pi l_{N}$ intersects $P$. By Lemma 1, applied with a change of scale, the number of such terms does not exceed $\left[1 / l_{N}\right]+2 p$. Hence Schwarz's inequality and (18) applied to (22) yield

$$
\begin{aligned}
& \int_{\sigma_{N}}\left|R_{N}(\omega)\right|^{2} d \omega \leqq\left\{\left[1 / l_{N}\right]+2 p\right\} \\
& \int_{\sigma_{N}} \sum_{r}\left|F\left(\omega-r 2 \pi l_{N}\right)\left\{H_{N}\left(\omega-r 2 \pi l_{N}\right)-\chi_{N}\left(\omega-r 2 \pi l_{N}\right)\right\}\right|^{2} d \omega \\
&=\left\{\left[1 / l_{N}\right]+2 p\right\} \int_{P}\left|F(\omega)\left\{H_{N}(\omega)-\chi_{N}(\omega)\right\}\right|^{2} d \omega \\
& \leqq \epsilon^{2}\left\{\left[1 / l_{N}\right]+2 p\right\}\|F\|^{2} .
\end{aligned}
$$

Also, by Schwarz's inequality applied to (20),

$$
\left|g_{N}\left(k / l_{N}\right)\right|^{2} \leqq \frac{\gamma_{N}}{2 \pi} \int_{\left|t-k / l_{N}\right|<1 / 2 l_{N}}|f(t)|^{2} d t
$$

Now, again by Lemma 1 , the number of points $k / l_{N}$ for which the interval $\left|t-k / l_{N}\right|<1 / 2 l_{N}$ intersects $Q$ does not exceed $\left[c l_{N}\right]+2 q$. As in Theorem 1 , we will require $f(t)$ to be orthogonal to the function $\overline{h_{N}\left(k / l_{N}-t\right)}$ for each such $k / l_{N}$; by (20) this is equivalent to the vanishing of $g_{N}\left(k / l_{N}\right)$. Thus by imposing no more than $\left[c l_{N}\right]+2 q$ orthogonality conditions on $f$, the sum on the left-hand side of (23) is extended over only those $k$ for which the interval $\left|t-k / l_{N}\right|<1 / 2 l_{N}$ lies entirely outside $Q$, so that by (25)

$$
\int_{\sigma_{N}}\left|F(\omega)+R_{N}(\omega)\right|^{2} d \omega \leqq \frac{\gamma_{N}}{2 \pi l_{N}} \int_{t \Subset Q}|f(t)|^{2} d t
$$

We expand the left-hand side of (26) and sum on $N$. We conclude that subjecting $f(t)$ to the requirements $\left(f, \psi_{i}\right)=0, i=1, \cdots, M$, where $\psi_{i}$ are fixed functions in $L^{2}$, each of the form $\overline{h_{N}\left(k / l_{N}-t\right)}$ for some $N$ and $k$, and $M \leqq \sum_{N}\left\{\left[c l_{N}\right]+2 q\right\} \leqq\left[c \sum_{N} l_{N}\right]+2 p q=[c]+2 p q$, ensures

$$
\begin{array}{r}
\sum_{N}\left\{\int_{\sigma_{N}}|F(\omega)|^{2} d \omega+\int_{\sigma_{N}}\left|R_{N}(\omega)\right|^{2} d \omega+2 \operatorname{Re} \int_{\sigma_{N}} F(\omega) \overline{R_{N}(\omega)} d \omega\right\} \\
\leqq \sum_{N} \frac{\gamma_{N}}{2 \pi l_{N}} \int_{t \notin Q}|f(t)|^{2} d t .
\end{array}
$$


Now $\sum_{N} \int_{\sigma_{N}}|F(\omega)|^{2} d \omega=\|F\|^{2}=\|f\|^{2}$. By Schwarz's inequality, (24), and the definition of $\epsilon$,

$$
\begin{aligned}
&\left|\sum_{N} 2 \operatorname{Re} \int_{\sigma_{N}} F(\omega) \overline{R_{N}(\omega)} d \omega\right| \\
& \quad \leqq 2 \sum_{N}\left\{\int_{\sigma_{N}}|F(\omega)|^{2} d \omega\right\}^{1 / 2}\left\{\int_{\sigma_{N}}\left|R_{N}(\omega)\right|^{2} d \omega\right\}^{1 / 2} \\
& \leqq 2\left\{\sum_{N} \int_{\sigma_{N}}|F(\omega)|^{2} d \omega\right\}^{1 / 2}\left\{\sum_{N} \int_{\sigma_{N}}\left|R_{N}(\omega)\right|^{2} d \omega\right\}^{1 / 2} \\
&\left.\leqq 2 \epsilon\|F\|^{2}\left\{2 p^{2}+\sum_{N} \mid 1 / l_{N}\right]\right\}^{1 / 2}=\|f\|^{2} / 2,
\end{aligned}
$$

so that from (27)

$$
\|f\|^{2} / 2 \leqq\left\{\int_{t \notin Q}|f(t)|^{2} d t\right\} \sum_{N} \frac{\gamma_{N}}{2 \pi l_{N}}=\left\{\|f\|^{2}-\|D f\|^{2}\right\} \sum_{N} \frac{\gamma_{N}}{2 \pi l_{N}} .
$$

Setting $J=\left(\sum_{N} \gamma_{N} / l_{N}-\pi\right) /\left(\sum_{N} \gamma_{N} / l_{N}\right)<1$ we may rewrite (29) as

$$
\|D f\|^{2} /\|f\|^{2} \leqq J
$$

whence by (19) $\lambda_{|c|+2 p q} \leqq J<1$. The constant $J$ depends on $P$ (normalized to have measure $2 \pi$ ) since the numbers $\gamma_{N}$ and $l_{N}$ do, but it does not depend on $Q$. Theorem 3 is established.

ThEOREM 4. Under the hypotheses of Theorem $3, \lambda_{[c]-2 p q} \geqq 1-J>0$.

Proof. We normalize $P$ to have measure $2 \pi$, as in Theorem 3 , and let $\sigma_{N}, l_{N}, \epsilon, \chi_{N}(\omega), h_{N}(t), \gamma_{N}$, and $J$ be as defined there. As in Theorem 2, we will base our proof on the modification of the Weyl-Courant lemma which asserts that

$$
\lambda_{\{c]-2 p q} \geqq \inf _{f \in \not(P) ; f \in S} \frac{\|D f\|^{2}}{\|f\|^{2}},
$$

where $S$ is a subspace of dimension at least $[c]-2 p q+1$.

For each $N=1, \cdots, p$ we let $I(N)$ be the set of integers $k$ for which the interval $\left|t-k / l_{N}\right|<1 / 2 l_{N}$ is contained in $Q$, and we define $S$ as the subspace generated by those members of $\mathscr{B}(P)$ whose Fourier transforms are the functions $\chi_{N}(\omega)\left(2 \pi l_{N}\right)^{-1 / 2} \exp \left(-i \omega k / l_{N}\right)$ with $k \in I(N)$. By Lemma 1 , applied with a change of scale, the number of integers in $I(N)$ exceeds $c l_{N}-2 q$, so that the total number of generators exceeds $\sum_{N=1}^{p}\left(c l_{N}-2 q\right)$ $=c-2 p q$ and, being an integer, is no smaller than $[c]-2 p q+1$. Since the generators form an orthonormal set, the dimension of $S$ is at least $[c]-2 p q+1$, as is required.

If $f \in S$, its Fourier transform, by definition, satisfies 


$$
F(\omega)=\sum_{k \in I(N)} a_{N, k} \exp \left(-i \omega k / l_{N}\right)\left(2 \pi l_{N}\right)^{-1 / 2}, \quad \omega \in \sigma_{N},
$$

and because the above exponentials are orthonormal over $\sigma_{N}$ we find

$$
\|F\|^{2}=\sum_{N=1}^{p} \sum_{k \in I(N)}\left|a_{N, k}\right|^{2} .
$$

Introducing the functions $g_{N}(x)$ of $(20)$ and $R_{N}(\omega)$ of (22) we obtain, as in (21),

$$
\begin{aligned}
\left(l_{N}\right)^{-1 / 2} g_{N}\left(k / l_{N}\right)= & \int_{\sigma_{N}} F(\omega)\left(2 \pi l_{N}\right)^{-1 / 2} \exp \left(i \omega k / l_{N}\right) d \omega \\
& +\int_{\sigma_{N}} R_{N}(\omega)\left(2 \pi l_{N}\right)^{-1 / 2} \exp \left(i \omega k / l_{N}\right) d \omega,
\end{aligned}
$$

and we denote by $c_{N, k}$ the last term in (32). Another appeal to the orthonormality of $\left(2 \pi l_{N}\right)^{-1 / 2} \exp \left(i \omega k / l_{N}\right)$ on $\sigma_{N}$, combined with (24), shows, for $k \in I(N)$,

$$
\begin{gathered}
\left(l_{N}\right)^{-1 / 2} g_{N}\left(k / l_{N}\right)=a_{N, k}+c_{N, k}, \\
\sum_{k \in I(N)}\left|c_{N, k}\right|^{2} \leqq \int_{\sigma_{N}}\left|R_{N}(\omega)\right|^{2} d \omega \leqq \epsilon^{2}\left\{\left[1 / l_{N}\right]+2 p\right\}\|F\|^{2} .
\end{gathered}
$$

Now from (33) and (25), together with the definition of $I(N)$,

$$
\sum_{k \in I(N)}\left|a_{N, k}+c_{N, k}\right|^{2}=l_{N}^{-1} \sum_{k \in I(N)}\left|g_{N}\left(k / l_{N}\right)\right|^{2} \leqq \frac{\gamma_{N}}{2 \pi l_{N}} \int_{t \in Q}|f(t)|^{2} d t .
$$

We expand the left-hand side of (35) and sum on $N$. Using Schwarz's inequality, (31), (32), Parseval's theorem, and the definition of $\epsilon$, we find, just as in (28),

$$
\left|\sum_{N=1}^{p} 2 \operatorname{Re} \sum_{k \in l(N)} a_{N, k} \overline{c_{N, k}}\right| \leqq\|f\|^{2} / 2,
$$

so that, by (31) and Parseval's theorem,

$$
\|f\|^{2} / 2 \leqq\|D f\|^{2} \sum_{N} \frac{\gamma_{N}}{2 \pi l_{N}}
$$

or

$$
\|D f\|^{2} /\|f\|^{2} \geqq 1-J,
$$

for all $f \in S$. By (30), $\lambda_{[c]-2 p q} \geqq 1-J>0$. Theorem 4 is established.

LemMa 2. Let $P$ be any fixed set of measure $2 \pi$, and $Q_{k}$ be a sequence of 
sets, each the union of $q$ intervals, and of total measure $c_{k} \rightarrow \infty$. Then the number of eigenvalues of $B_{P} D_{Q_{k}} B_{P}$ contained in any fixed subinterval of the unit interval cannot remain bounded as $k \rightarrow \infty$.

Proof. To simplify notation we will write $B$ and $D_{k}$ instead of $B_{P}$ and $D_{Q_{k}}$, respectively.

M. Rosenblum has shown [to appear] that the Wiener-Hopf operator $A f=(2 \pi)^{-1 / 2} \mathcal{S}_{0}^{\infty} h(t-x) f(x) d x, t \geqq 0$, on $L^{2}(0, \infty)$ may be taken into a Toeplitz transformation by a unitary mapping. If the Fourier transform $H(\omega)$ of $h(t)$ is bounded, the spectrum of $A$ may then be determined by the results of [1]. In particular, when $H(\omega)$ is real, this method shows the spectrum of $A$ to consist of all numbers between ess sup $H(\omega)$ and ess inf $H(\omega)$. I am indebted to $\mathrm{H}$. Widom for suggesting this argument, on which our proof will be based.

Letting $S$ be the set $t \geqq 0, D$ be the projection $D_{S}$, and $H(\omega)$ be the characteristic function of $P$, the operator $D B D$ coincides with $A$. Thus the spectrum of $D B D$ consists of all $0 \leqq \lambda \leqq 1$.

We now show that $D B D$ and $B D B$ have the same spectra. For by definition, if $0<\lambda<1$ is not in the spectrum of $B D B$, the operator $B D B-\lambda B$ has an inverse bounded by some $M$. Then from the equation $D B D f-\lambda D f$ $=g$, using the idempotency of projections and their boundedness, we obtain

$$
\begin{aligned}
|\lambda|\|D f\|-\|g\| \leqq\|\lambda D f+g\| & =\|D B D f\| \leqq\|B D f\| \\
& =\left\|(B D B-\lambda B)^{-1} B g\right\| \leqq M\|g\| .
\end{aligned}
$$

But since $D f=(D B D-\lambda D)^{-1} g$, this shows $D B D-\lambda D$ to have an inverse bounded by $(M+1) /|\lambda|$, contradicting the fact that $\lambda$ is in the spectrum of $D B D$. Since the spectrum of $B D B$ is a closed subset of the unit interval, it consists of all $0 \leqq \lambda \leqq 1$.

Next let $\tau_{i}(k), i=1, \cdots, q$ be the $(i)$ th interval of $Q_{k}$, counted from the left. Let $r$ be the least integer for which the length of $\tau_{r}(k)$ becomes unbounded as $k \rightarrow \infty ; 1 \leqq r \leqq q$, since by assumption $c_{k} \rightarrow \infty$. Because the eigenvalues of $B D_{k} B$ are not affected by a translation of $Q_{k}$, we may suppose that the left-hand endpoint of $\tau_{r}(k)$ coincides with the origin. By choosing a suitable subsequence of the sets $Q_{k}$, we may also suppose that each $\tau_{i}(k)$, $i<r$, converges to a limit (possibly to infinity). Let $S^{\prime}$ be the set of all points on $-\infty<t<0$ which are limits of points in $\tau_{i}(k), i<r$, and let $D^{\prime}$ denote the projection $D_{S^{\prime}}$. Then for any fixed $\phi \in L^{2}$,

$$
\left\|\left(D+D^{\prime}\right) \phi-D_{k} \phi\right\| \rightarrow 0
$$

as $k \rightarrow \infty$.

Since by definition of $r$ the lengths of $\tau_{i}(k), i<r$, are bounded, $S^{\prime}$ has finite measure, so the operator $B D^{\prime} B$ is completely continuous. By a theorem 
of Weyl [5, p. 367], the addition of such an operator to the bounded selfadjoint $B D B$ does not change any limit point of the spectrum. Thus the spectrum of $B\left(D+D^{\prime}\right) B$ contains all $0<\lambda<1$. Consequently given $\lambda$, $0<\lambda<1$, and $\epsilon>0$, there exists a function $B f_{\epsilon}$, with $\left\|B f_{\epsilon}\right\|=1$ and

$$
\left\|B\left(D+D^{\prime}\right) B f_{\epsilon}-\lambda B f_{\epsilon}\right\|<\epsilon / 2 \text {. }
$$

Now by (37) we may choose $k_{0}$ so that for all $k>k_{0},\left\|\left(D+D^{\prime}\right) B f_{\epsilon}-D_{k} B f_{\epsilon}\right\|$ $<\epsilon / 2$. Since $\|B\| \leqq 1$, this implies $\left\|B\left(D+D^{\prime}\right) B f_{\epsilon}-B D_{k} B f_{\epsilon}\right\|<\epsilon / 2$, and combining this with (38) yields $\left\|B D_{k} B f_{\epsilon}-\lambda B f_{\epsilon}\right\| \leqq \epsilon$. Then by the triangle inequality

$$
\left\|B D_{k} B f_{\epsilon}\right\| \geqq \lambda-\epsilon,
$$

and since $B D_{k} B$ is bounded by 1 ,

$$
\left\|\left(B D_{k} B\right)^{2} f_{\epsilon}-\lambda B D_{k} B f_{\epsilon}\right\| \leqq\left\|B D_{k} B f_{\epsilon}-\lambda f_{\epsilon}\right\| \leqq \epsilon .
$$

But by $\left[5\right.$, p. 234] the eigenfunctions $\phi_{i}^{(k)}$ of $B D_{k} B$ are sufficient to expand any element in its range. Thus we may write

$$
B D_{k} B f_{\epsilon}=\sum_{i} a_{i} \phi_{i}^{(k)}
$$

whence by (39)

$$
(\lambda-\epsilon)^{2} \leqq\left\|B D_{k} B f_{\epsilon}\right\|^{2}=\sum_{i}\left|a_{i}\right|^{2}
$$

and

$$
\left(B D_{k} B\right)^{2} f_{\epsilon}=\sum_{i} a_{i} B D_{k} B \phi_{i}^{(k)}=\sum_{i} a_{i} \lambda_{i}^{(k)} \phi_{i}^{(k)},
$$

where $\lambda_{i}^{(k)}$ is the (i) th eigenvalue of $B D_{k} B$. Introducing (41) and (43) into (40) yields by (42)

$$
\epsilon^{2} \geqq \sum_{i}\left|a_{i}\right|^{2}\left|\lambda_{i}^{(k)}-\lambda\right|^{2} \geqq \inf _{i}\left|\lambda_{i}^{(k)}-\lambda\right|^{2}(\lambda-\epsilon)^{2} .
$$

Choosing $\epsilon$ sufficiently small, we conclude that every neighborhood of $\lambda$ will contain an eigenvalue of $B D_{k} B$ for all $k>k_{0}$.

To complete the proof of the lemma, given any subinterval $I$ of the unit interval, and any integer $N$, we divide $I$ into $N$ disjoint subintervals. By the above, each of these subintervals will contain an eigenvalue of $B D_{k} B$ for all $k$ sufficiently large. Thus the number of eigenvalues of $B D_{k} B$ contained in $I$ cannot remain bounded as $k \rightarrow \infty$. Lemma 2 is established.

Corollary 2. Under the hypotheses of Theorem 3, with any fixed integer $N$,

$$
\lambda_{[c]-N} \leqq J_{1}<1,
$$

where $J_{1}$ is a constant depending on $P$ (suitably normalized), $q$, and $N$, but not on $Q$. 
Proof. We will argue by contradiction. Accordingly, let us suppose that for a given $P$, normalized as in Theorem 3 to have measure $2 \pi$, there exists a sequence of sets $S_{k}$, each the union of $q$ intervals and of total measure $c_{k}$, for which $\lambda_{\left|c_{k}\right|-N} \rightarrow 1$. To simplify notation, let us denote the projections $B_{P}$ and $D_{S_{k}}$ by $B$ and $D_{k}$ respectively, and the eigenvalue $\lambda_{\left[c_{k}\right]-N}$ of $B D_{k} B$ by $\lambda_{k}^{*}$.

By Theorem 3, $\lambda_{\left|c_{k}\right|+2 p q} \leqq J<1$, and we are assuming $\lambda_{k}^{*} \rightarrow 1$. Thus for all $K$ sufficiently large, the interval $J \leqq x \leqq(J+1) / 2$ will contain no more than $2 p q+N$ eigenvalues of $B D_{k} B$. We conclude by Lemma 2 that the measures $c_{k}$ of $S_{k}$ must be bounded: $c_{k}<C$.

Now let $\psi^{(k)}(t)$ be the eigenfunction of $B D_{k} B$ corresponding to $\lambda_{k}^{*}$, normalized so that $\left\|\psi^{(k)}\right\|=1$. We find, as in the transformations leading to (2)

$$
\int_{S_{k}}\left|\psi^{(k)}(t)\right|^{2} d t=\lambda_{k}^{*} \rightarrow 1 .
$$

Consequently, for one of the $q$ subintervals of $S_{k}$, which we denote by $\tau(k)$,

$$
\int_{\tau(k)}\left|\psi^{(k)}(t)\right|^{2} d t>1 / 2 q .
$$

Since the eigenvalues of $B D_{k} B$ are not affected by a translation of $S_{k}$ along the $t$-axis, we may suppose that the left-hand endpoint of $\tau(k)$ coincides with the origin. As we showed in the preliminary remarks, it follows from the normalization $\left\|\psi^{(k)}\right\|=1$ of $\psi^{(k)} \in \mathscr{B}(P)$ that the functions $\psi^{(k)}(t)$ form a uniformly bounded family of analytic functions in any horizontal strip including the real axis, thus a normal family there. We may therefore suppose that

$$
\psi^{(k)}(t) \rightarrow \psi(t),
$$

uniformly on compact subsets of the $t$-axis, for this may always be ensured by choosing a suitable subsequence; $\psi(t)$ is then also analytic. For the same reason we may suppose that each subinterval of $S_{k}$ converges to a limit (possibly to infinity). Since $c_{k}<C$, this limit of $S_{k}$ has finite measure, so its complement includes some finite interval $I$; thus there exists $k_{0}$ such that $I$ is disjoint from every $S_{k}, k>k_{0}$. Then for $k>k_{0}$, by the normalization of $\psi^{(k)}$ and (44),

$$
\begin{aligned}
\int_{I}\left|\psi^{(k)}(t)\right|^{2} d t \leqq \int_{t \notin S_{k}}\left|\psi^{(k)}(t)\right|^{2} d t & =1-\int_{S_{k}}\left|\psi^{(k)}(t)\right|^{2} d t \\
& =1-\lambda_{k}^{*} \rightarrow 0,
\end{aligned}
$$

whence by (46)

$$
\int_{I}|\psi(t)|^{2} d t=0
$$


or $\psi(t)=0, t \in I$. The analyticity of $\psi(t)$ then implies $\psi(t) \equiv 0$. On the other hand, let $0<t<\alpha$ be the limit interval of $\tau(k)$. Since the functions $\psi^{(k)}(t)$ are uniformly bounded, (45) implies $\alpha \neq 0$, and $c_{k}<C$ implies $\alpha<C$. We may then again apply (46) on the finite interval $0 \leqq t \leqq \alpha$ to conclude $\int_{0}^{\alpha}|\psi(t)|^{2}>1 / 2 q$, whence $\psi(t) \not \equiv 0$, and we have reached a contradiction. Corollary 2 is established.

CoRollary 3. Under the hypotheses of Theorem 3, with any fixed integer $N$ and $c \geqq 1$,

$$
\lambda_{[c]+N} \geqq J_{2}>0,
$$

where $J_{2}$ is a constant depending on $P$ (suitably normalized), $q$, and $N$, but not on $Q$.

Proof. The restriction $c \geqq 1$ is necessary, since as $c \rightarrow 0$ every eigenvalue approaches 0 . As in the proof of Corollary 2, we will argue by contradiction. Accordingly, let us suppose that for a given $P$, normalized as in Theorem 3 to have measure $2 \pi$, there exists a sequence of sets $S_{k}$, each the union of $q$ intervals and of total measure $c_{k}$, for which $\lambda_{\left|c_{k}\right|+N} \rightarrow 0$. To simplify notation, we denote the projections $B_{P}$ and $D_{S_{k}}$ by $B$ and $D_{k}$ respectively, and the eigenvalue $\lambda_{\left[c_{k}\right]+N}$ of $B D_{k} B$ by $\lambda_{k}^{\prime}$.

By Theorem $4, \lambda_{\left|c_{k}\right|-2 p q} \geqq J^{\prime}>0$, and we are assuming $\lambda_{k}^{\prime} \rightarrow 0$. Thus for all $k$ sufficiently large, the interval $J^{\prime} / 2 \leqq x \leqq J^{\prime}$ will contain no more than $2 p q+N$ eigenvalues of $B D_{k} B$. By Lemma 2, the measures $c_{k}$ of $S_{k}$ must be bounded: $c_{k}<C$. Then $\left[c_{k}\right]+N<[C]+N$ so that

$$
\lambda_{k}^{\prime} \geqq \lambda_{[C]+N}^{(k)},
$$

where $\lambda_{[C]+N}^{(k)}$ is the eigenvalue $\lambda_{[C]+N}$ of $B D_{k} B$. Since $S_{k}$ has total measure $c_{k} \geqq 1$, at least one of its $q$ subintervals will have measure exceeding $1 / 2 q$. Because the eigenvalues of $B D_{k} B$ are not affected by a translation of $S_{k}$, we may suppose $S_{k}$ to include the interval $I: 0 \leqq t \leqq 1 / 2 q$. Letting $T_{k}$ denote the remainder of $S_{k}$, we may write $B D_{k} B=B D_{I} B+B D_{T_{k}} B$. As we showed in the preliminary remarks, the latter operator is positive, thus by an application of the Weyl-Courant lemma [5, p. 239],

$$
\lambda_{[C]+N}^{(k)} \geqq \lambda_{[C]+N}^{(I)},
$$

where $\lambda_{[C]+N}^{(I)}$ is the eigenvalue $\lambda_{[C]+N}$ of $B D_{I} B$, and hence is positive. Combining the above with (47) yields $\lambda_{k}^{\prime} \geqq \lambda_{[C]+N}^{(I)}>0$, and since the right-hand quantity is independent of $k$, this contradicts our assumption that $\lambda_{k}^{\prime} \rightarrow 0$. Corollary 3 is established.

\section{BibLIOGRAPHY}

1. A. Calderon, F. Spitzer, and H. Widom, Inversion of Toeplitz matrices, Illinois J. Math. 3 (1959), 490-498. 
2. W. H. J. Fuchs, On the eigenvalues of an integral equation, Notices Amer. Math. Soc. 10 (1963), 352.

3. H. J. Landau and H. O. Pollak, Prolate spheroidal wave functions, Fourier analysis and uncertainty. II, Bell System Tech. J. 40 (1961), 65-84.

4. - Prolate spheroidal wave functions, Fourier analysis and uncertainty. III, Bell System Tech. J. 41 (1962), 1295-1336.

5. F. Riesz and B. Sz-Nagy, Functional analysis, Ungar, New York, 1955.

6. D. Slepian and H. O. Pollak, Prolate spheroidal wave functions, Fourier analysis and uncertainty. I, Bell System Tech. J. 40 (1961) 43-64.

7. H. Widom, Extreme eigenvalues of $N$-dimensional convolution operators, Trans. Amer. Math. Soc. 106 (1963), 391-414.

BELL TELEPHONE LABORATORIES, INCORPORATED, Murray Hills, New Jersey 